\title{
A Mechanical Rectifier for the Measurement of Small, Commercial-Frequency, Alternating Potentials
}

\author{
Carsten C. Steffens and Richard C. Tolman, Gates Chemical Laboratory, California Institute of Technology
}

(Received April 25, 1934)

\begin{abstract}
A mechanical rectifier for use at commercial frequencies has been constructed. Accidental electromotive forces have been so far eliminated that it can be used with a sensitive directcurrent galvanometer as a detecting instrument. Four galvanometers have been tried and the results are described. With the most sensitive galvanometers used, the sensitivities of the system at 50 cycles were 0.05 microvolt (without the critical damping resistance), and 40 micromicroamperes per millimeter at one meter.
\end{abstract}

\section{INTRODUCTION}

$\mathrm{H}^{1}$ IGH sensitivity is more difficult to attain in the measurement of alternating potentials than in the measurement of directcurrent potentials. Instruments sensitive to less than one microvolt at fifty cycles are either expensive or sensitive to changes in the electrical network to which they are connected, or both, compared with the analogous instruments for direct potentials. In an attempt to remove these impediments, we have designed and tested a mechanical rectifier for use with high-sensitivity direct-current galvanometers.

Mechanical rectifiers of the type of synchronous commutators have long been used in commercial practice. Two inherent difficulties, however, prevent their use with sensitive galvanometers. The first of these is the friction and varying resistance at the brush contacts. This causes varying and unknown electromotive forces in the commutator itself as well as fluctuations in the current being rectified. The second difficulty is due to the potential difference between the two ends of a commutator bar when that bar is cutting the earth's magnetic field.

Somewhat successful attempts have already been made to overcome these difficulties. Thus Dannatt and Holt $\mathbf{t}^{1}$ have described an improved commutator with brushes of special graphite in specially designed holders. This could be satisfactorily used in connection with a milliammeter.

As an alternative solution of the problem which is somewhat similar to the method that

${ }^{1}$ C. Dannatt and N. Holt, World Power 7, 241 (1927). we have employed, Sharp and Crawford ${ }^{2}$ have designed and tested a synchronous reversing key, which avoids rotating parts that would cut the earth's field, and is provided with platinum contact points to reduce variable effects. This could be satisfactorily used in connection with a microammeter. As far as we know, however, no rectifier suitable for use with really high-sensitivity galvanometers has hitherto been described in the literature.

\section{Description of Rectifier}

The main ideas involved in the apparatus to be described in this note are to use a set of contact points, made of the same material as the rest of the circuit (copper) so as to avoid thermal electromotive forces, designed with large volume and large contact surface so as to dissipate heat and iron out local surface effects, and operated by a rotating cam in such a way as to make contact without slipping as is done by a galvanometer key. Phase adjustability is obtained by mounting the contact points on a rotatable face plate.

The details of the design adopted are shown in Figs. 1 and 2. The bronze cam $A$ turns in a steel bushing $B$. This bushing is free to slide back and forth in the bronze plate $C$, but the vertical component (as Fig. 1 is printed) of the motion of $B$ is transmitted to $C$ which slides on steel rods $D$, supported by the brass

${ }^{2}$ Sharp and Crawford, Proc. A.I.E.E. 29, Part 2, 1518 (1910). 


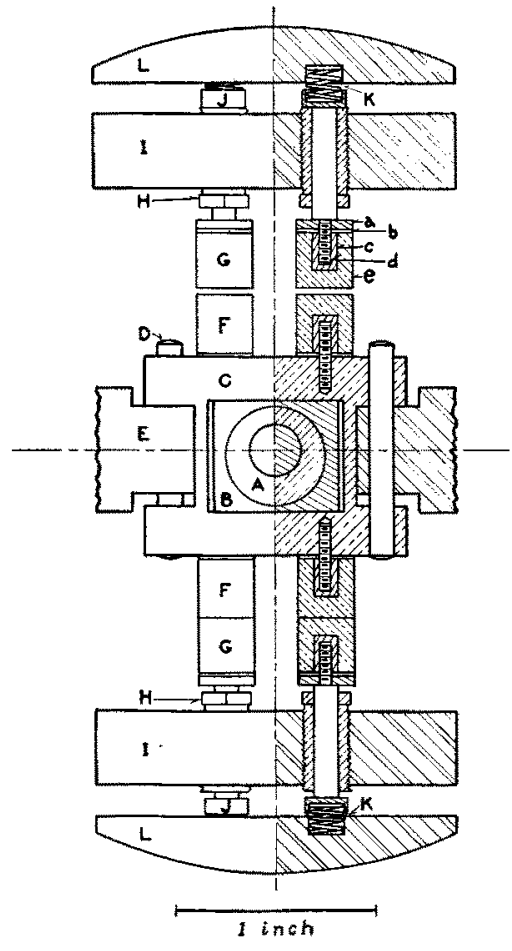

FIG. 1.

blocks $E$. One set of contact points $F$ are attached to the block $C$. The other set of points $G$ are attached to steel rods whose outer ends $J$ are enlarged and hollow to take spiral steel springs, $K$. The outer ends of the springs rest in holes in the brass blocks $L$. The rods carrying the contact points slide in bronze bushings with hexagonal heads, $H$, which are screwed through the brass blocks $I$. The brass blocks, $E, I$ and $L$, are screwed and pinned to a brass face plate which is rotatable about the shaft. The steel bushing $B$ is tapered on the edges bearing on $C$ and is held in place by a steel plate fastened to the face of $B$ by four screws. This plate covers $A, B$, and the end of the shaft in the photograph (Fig. 2).

The construction was sufficiently accurate so that no play could be felt in the chain of bearings between the shaft and the contact points $F$, nor any looseness in the contact points $G$.

The cam has a throw of $1 / 40$ inch. (Exaggerated in Fig. 1.)

The contacts are made by pressing a Redmanol plug, $c$, into a hole in the copper cylinder $e$. The plug $c$ is tapped to take the threaded end $d$

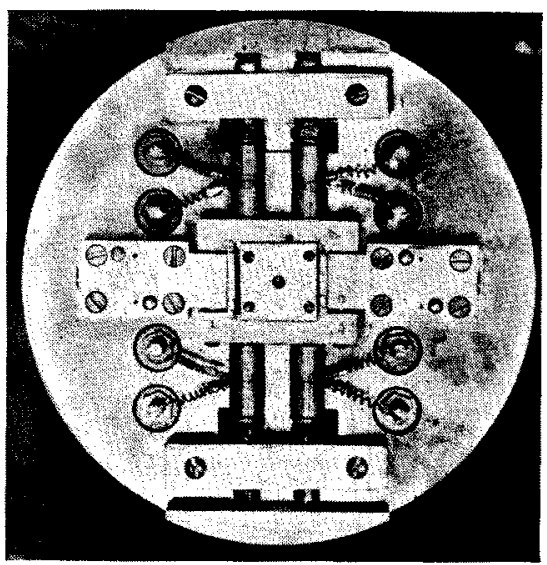

FIG. 2. Front view of the rectifer.

of the rod $J$ or for attachment to the plate $C$, as the case may be. The insulation is completed by a mica disk, $b$. The assembly is supported, in the case of the outer contact points $G$, by the steel disk, $a$.

Coiled copper wires are soldered to copper pins driven into the copper heads of the contact points. The outer ends of the wires are soldered to copper binding posts set in Redmanol plugs pressed into holes through the face plate. There is nothing but copper in the electrical circuit.

A $1 / 4$ horsepower synchronous motor (somewhat larger than necessary) drives the rectifier. The motor is enclosed in a shield made of eighteen layers of alternate soft iron and copper sheet each 0.018 inch thick, the iron being brought out at the edges and bolted to give a continuous magnetic circuit. A flexible coupling with a fiber disk connects the rectifier to, and insulates it from, the motor.

\section{Adjustment of the Rectifier}

The positions of the contact points are adjusted visually and tactually by turning the bushings $H$ until contact seems to occur in the middle of the stroke. A constant error of not greater than 5 percent in the reading of an attached instrument may be expected from adjustments made in this way. If greater precision is desired, a dry cell may be so connected to the rectifier that the alternating-current output feeds into a millivoltmeter, and the contacts adjusted to give zero deflection. The contact 
points must be cleaned with alcohol occasionally.

To make the phase adjustment, alternating current of the phase to be used is fed through the rectifier into the measuring instrument and the face plate rotated until zero deflection is obtained. The face plate is then set 90 degrees from this position.

\section{Behavior of the Rectifier with Various Galvanometers}

The behavior of the rectifier with four galvanometers of different characteristics has been studied. With the more sensitive galvanometers, all wire loops on the input side had to be reduced to the smallest practicable dimensions and any motors or transformers in the vicinity had to be shielded, as the system is very sensitive to alternating fields of the frequency to which it is tuned. It was also necessary to ground the face plate.

The galvanometers used were all Leeds and Northrup instruments. Sensitivities are given in effective currents or potentials per scale division, or per millimeter at one meter without critical damping resistance.

With a pointer galvanometer $2320-d$, coil resistance $1000 \mathrm{ohms}$, period 3 seconds, directcurrent sensitivity 0.5 microampere, the alternating-current sensitivity is 0.6 microampere. The deflections are linear with the current, steady, and reverse exactly if the face plate is rotated through 180 degrees. The motor need not be shielded with this instrument.

With a type $P$ reflecting galvanometer 2239-a, coil resistance 124 ohms, period 10 seconds, direct-current sensitivity 0.011 microampere, the alternating-current sensitivity is 0.018 microampere. The deflections are linear with the current, steady, and reverse exactly if the face plate is rotated through 180 degrees. Without the motor shield, the zero readings on the galvanometer may vary by 3 millimeters when the face plate is rotated.
With a type $H S$ reflecting galvanometer 2285-a, coil resistance 18.4 ohms, period 8.3 seconds, direct-current sensitivity 0.033 microvolt, the alternating-current sensitivity is 0.048 microvolt. The deflections are approximately linear with the current and are steady to about one millimeter. The motor shield is essential and even with it, the zero readings may vary by 5 millimeters when the face plate is rotated.

With a type $H S$ reflecting galvanometer 2285-f, coil resistance 915 ohms, period 21.1 seconds, direct-current sensitivity 28 . micromicroamperes, the alternating-current sensitivity is 33. micromicroamperes. The deflections are approximately linear with the current and are as steady as in direct current measurements. There may be a variation of some 5 centimeters in the zero reading when the face plate is rotated with the motor shielded. This variation is affected by such things as turning the room lights off and on.

\section{Discussion}

The use of this rectifier together with a directcurrent galvanometer has the advantages over the vibration galvanometer of greater sensitivity and of automatic tuning to the proper frequency, but requires a source of power, either mechanical or electrical, of the same frequency as that of the effect to be measured. Over the alternatingcurrent galvanometer, there are the advantages of independence of the circuit constants (except insofar as they affect the damping), of the possibility of using mechanical rather than electrical power, and of using a standard directcurrent galvanometer rather than an expensive, separate instrument. Measurements of phase are difficult with the alternating-current galvanometer, and impossible with the vibration galvanometer alone, but are quite simple with this rectifier.

We are much indebted to Mr. Julius Pearson in connection with the mechanical construction of the rectifier. 